\title{
UNA EXPERIENCIA INCLUSIVA \\ DE APRENDIZAJE COOPERATIVO: FOMENTANDO HABILIDADES PARA EL EMPLEO EN LA UNIVERSIDAD
}

\section{An inclusive experience of cooperative learning: promotion of skills for employment in the university}

Irina Sherezade Castillo Reche

Universidad de Murcia

irinasherezade.castillo@um.es

Belén SuÁrez Lantarón

Universidad de Murcia

Recepción: 17 de septiembre de 2019

Aceptación definitiva: 17 de marzo de 2020

Resumen: En el artículo se describe una experiencia de aprendizaje cooperativo en la que han participado alumnado de Educación Social y alumnado con discapacidad intelectual del programa Todos Somos Campus de la Universidad de Murcia. Dicha experiencia ha sido analizada con el objetivo de conocer los beneficios y satisfacción de todos los participantes. Para ello, se ha utilizado una metodología mixta en la que la recogida de información se ha realizado con diferentes instrumentos. Las posibilidades y ventajas de ambos enfoques permiten una triangulación de la información durante todo el proceso. Los resultados obtenidos nos indican que la experiencia ha resultado motivadora para todos los participantes, quienes además han desarrollado numerosas habilidades y competencias, lo que ha conllevado una alta satisfacción. Todo ello nos lleva a concluir que la presencia de este tipo de experiencias no beneficia solamente a los estudiantes con discapacidad, sino a todos aquellos que participan en ellos y que el uso de metodologías de aprendizaje cooperativo promueve que los alumnos sean protagonistas de su propio aprendizaje y, por tanto, se realicen aprendizajes más significativos.

Palabras Clave: discapacidad intelectual; educación social; universidad; educación inclusiva; metodologías activas. 
АвsтRAст: This paper describes a cooperative learning experience in which Social Education students and students with intellectual disabilities attending the "We're all Campus" Program of the University of Murcia have participated. This experience has been reviewed in order to know the benefits and satisfaction of all participants. To this end, a mixed methodology has been used in which the collection of information has been carried out with different instruments. The possibilities and advantages of both approaches allow triangulation of information throughout the process. The results obtained indicate that the experience has been motivating for all participants, who have also developed numerous skills and competencies, which has led to high satisfaction. All of this leads us to conclude that the presence of these types of experiences does not only benefit students with disabilities but also all those who participate in them. The use of cooperative learning methodologies promotes that students are protagonists of their own learning and therefore, more meaningful learning takes place.

KEY WORDS: intellectual disability; social education; university; inclusive education; active methodologies.

\section{Introducción}

A Universidad DE Murcia (en adelante UM) ha puesto en marcha, a través del Vicerrectorado de Estudiantes, el Vicerrectorado de Empleo, Emprendimiento y Sociedad y el Vicerrectorado de Estudios, el Programa Todos Somos Campus (TSC), un programa que se financia con Fondos Sociales Europeos y con la participación de la Fundación ONCE. Los Servicios implicados en dicho programa son el de Atención a la Diversidad y Voluntariado (ADyV) y el Centro de Orientación e Información para el Empleo (COIE). Se trata de un programa que pretende brindar a los jóvenes con discapacidad intelectual la oportunidad de ir a la Universidad y participar en la vida activa universitaria al mismo tiempo que los prepara para una adecuada inserción laboral. Si bien no es objeto de este artículo desarrollar una exposición minuciosa de las características del Programa señalado, es necesario contextualizarlo ya que es un aspecto fundamental de este. Los jóvenes estudiantes con discapacidad asisten a las aulas de la universidad durante dos cursos académicos para mejorar sus habilidades en relación al empleo y así posibilitar una mejora de la inclusión laboral de este colectivo. Lo que permite el desarrollo de competencias fundamentales para el empleo como la responsabilidad, la autonomía y la motivación (Izuzquiza y Rodríguez, 2016). Asimismo, con el impulso del programa se da la oportunidad a los alumnos con discapacidad intelectual (se utiliza este término siguiendo el usado por Plena inclusión, organización que representa en España a las personas con discapacidad intelectual o del desarrollo), para que se relacionen con otros miembros de la comunidad universitaria de modo formal o informal (en las bibliotecas, las cafeterías, aulario, y la vida en el campus en general).

En este artículo se expone una experiencia de relación formal entre los alumnos de 2. ${ }^{\circ}$ de Educación Social que cursan la asignatura "Procesos y Condiciones para el Desarrollo de Proyectos” y los alumnos del Programa TSC en la que ambos 
han aprendido juntos a través del trabajo coordinado y organizado mediante grupos de aprendizaje cooperativo.

La presencia de este tipo de experiencias no beneficia solamente a los estudiantes con discapacidad quienes, por el simple hecho de asistir físicamente al entorno universitario, obtienen beneficios y ven incrementadas sus posibilidades de futuro laboral; sino que enriquece a todo el contexto universitario, mejorando la percepción y actitudes hacia la discapacidad intelectual y permitiendo el desarrollo de valores como equidad, igualdad y respeto (Cerrillo, Izuzquiza y Egido, 2013; Izuzquiza y Rodríguez, 2016). Contribuyendo de este modo a la construcción de una sociedad más igualitaria y justa y, por ende, de una institución universitaria mejor (Hart, Grigal, Sax, Martínez y Will, 2006).

\section{Marco teórico: aprendizaje cooperativo y educación inclusiva}

El cambio hacia un modelo educativo que emerja desde los nuevos planteamientos del Espacio Europeo de Educación Superior requiere el uso de metodologías o estrategias de enseñanza-aprendizaje en las que los alumnos han de tener el protagonismo en la adquisición de conocimientos, competencias y habilidades (Florido, Jiménez y Santana, 2011; Palomares, 2009; Ochoa, Pérez y Salinas, 2018), actuando el docente como guía de dicho proceso, adecuando y dinamizado los contextos de aprendizaje a las necesidades del alumno (García-Ruiz, Guerra, González y Álvarez, 2010).

La metodología de aprendizaje cooperativo implica al alumnado en su formación, al mismo tiempo que favorece la construcción colectiva del conocimiento, ya que parte de la organización del aula en pequeños grupos heterogéneos, donde los alumnos profundizan en tareas de aprendizaje de forma coordinada y que tienen una meta común implicando no solo su aprendizaje, sino el del resto de sus compañeros (Domingo, 2010; Slavin, 2014). Metodologías como el aprendizaje cooperativo generan aprendizajes más profundos, significativos y duraderos mejorando los resultados académicos de los alumnos (Gutiérrez-Fresneda, 2017; Torrego y Negro, 2012), ya que facilitan la conexión entre teórica y práctica, promoviendo, de ese modo, un aprendizaje significativo, útil, relevante y motivador.

Esta metodología ha sido objeto de multitud de investigaciones debido a su efectividad en relación a los logros académicos y por sus posibilidades de desarrollo afectivo, cognitivo, social y ciudadano (Trujillo y Ariza, 2006; Domingo 2010; Salmerón, 2010) ya que permite, entre otros, el perfeccionamiento de competencias tanto emocionales como profesionales y sociales (Johnson y Johnson, 2009; 2014).

Se adquieren, a través del aprendizaje cooperativo, por tanto, competencias transversales que promueven la comunicación eficaz de las ideas, la resolución de conflictos, la adaptación a los cambios, el análisis crítico y la toma de decisiones (Estrada, Monferrer y Moliner, 2016); se aumentan las capacidades de trabajo en equipo, la sensibilidad social, la autonomía y la empatía hacia los demás. Mejorando así la convivencia, el respeto por las opiniones y creencias de otros, la búsqueda del bien común, 
la cooperación y la solidaridad (Azorín, 2018; León del Barco, Mendo, Felipe, Polo y Fajardo, 2017).

Estas metodologías cobran especial relevancia cuando hablamos de diversidad, pues resulta difícil pensar en una educación inclusiva sin que en ella existan prácticas colaborativas (Azorín, 2018). Se trata de una metodología beneficiosa para estudiantes con discapacidad ya que está considerada como una herramienta metodológica capaz de dar respuesta a diferentes necesidades que presentan los discentes. Por tanto, favorece la inclusión educativa y social de los estudiantes con capacidades diferentes (Johnson y Johnson, 2014). El trabajo cooperativo permite entender que toda persona puede aportar en el proceso de aprendizaje (Lata y Castro, 2016), sean cuales sean sus necesidades educativas (Pujolás, 2012). Resulta difícil imaginar una educación inclusiva sin trabajo cooperativo (Riera, 2011).

\section{Metodología}

Se muestra un estudio descriptivo acerca de una experiencia educativa, con una metodología eminentemente cualitativa dada la naturaleza de este. Se utilizan diferentes modos de indagación con la finalidad de dar una respuesta ajustada al objetivo de investigación y obtener una perspectiva que tienda hacia una visión holística y que permita describir la experiencia educativa de forma global.

\subsection{Objetivo}

Conocer la valoración y beneficios de una experiencia de aprendizaje cooperativo entre jóvenes con discapacidad intelectual y alumnado de 2. ${ }^{\circ}$ de Educación Social.

\subsection{Contexto y participantes}

La experiencia se ha desarrollado en la Facultad de Educación de la Universidad de Murcia y ha contado con la participación de:

- 30 alumnos de $2 .^{\circ}$ curso de Educación Social adscritos a la asignatura "Procesos y Condiciones para el Desarrollo de Proyectos".

- 15 estudiantes con discapacidad intelectual del 1. ${ }^{\text {er }}$ curso del Programa Todos Somos Campus.

- Tres docentes del Departamento de Didáctica y Organización Escolar que imparten docencia en el grado de Educación Social y desarrollan el módulo de habilidades emocionales en la empresa y entrenamiento cognitivo para el empleo en el Programa Todos Somos Campus.

- 1 técnico de apoyo que acompaña siempre en el aula al alumnado de Todos Somos Campus 


\subsection{Desarrollo de la experiencia}

\subsubsection{Origen y contextualización de la experiencia}

La experiencia educativa surge a partir de la idea de dos personas que imparten docencia en los grupos de TSC y Educación Social. Observan que algunos contenidos y competencias a desarrollar son comunes y que la interacción de ambos puede favorecer su aprendizaje. Los alumnos de TSC han de desarrollar habilidades para el empleo y los alumnos de Educación Social han de implementar un proyecto que mejore la empleabilidad de los educadores sociales; desde el programa TSC llevan trabajando el desarrollo de habilidades para el empleo durante meses, por lo que pueden ser de gran ayuda para el grupo de educadores sociales. Además, de este modo el alumnado de TSC tiene la posibilidad de desarrollar y ampliar los conocimientos adquiridos hasta entonces. Se decide utilizar la metodología de aprendizaje cooperativo dada la naturaleza de los contenidos que han de trabajar los estudiantes ya que fomenta el desarrollo de algunas de las competencias que se busca adquirir (trabajo en equipo, hablar en público, etc.).

\subsubsection{Competencias y contenidos}

En la Tabla 1 quedan reflejados los contenidos y competencias que, según las guías docentes, han de desarrollar los alumnos en sus respectivas asignaturas y que se relacionan directamente con los desarrollados en esta experiencia. Aunque dichos contenidos y competencias son específicos de cada grupo, han sido puestos en práctica de forma conjunta, desarrollándose así aprendizajes por ambos grupos de alumnos relativos a todas las competencias.

\begin{tabular}{|l|l|l|}
\hline \multicolumn{2}{|c|}{ TABla 1. Contenidos trabajados y competencias a adquirir por el alumnado } \\
\hline Contenidos & $\begin{array}{l}\text { Todos Somos Campus } \\
\text { Habilidades emocionales } \\
\text { en la empresa. } \\
\text { Entrenamiento cognitivo } \\
\text { para el empleo. }\end{array}$ & $\begin{array}{l}\text { 2. }{ }^{\text {de }} \text { Educación Social } \\
\text { Desarrollo de proyectos de intervención. } \\
\text { Diseminación y utilización de conocimien- } \\
\text { to. Del diseño a la puesta en práctica. Facto- } \\
\text { res y procesos implicados. Implementación } \\
\text { de proyectos. Noción y caracterización. En- } \\
\text { foques de implementación. }\end{array}$ \\
\hline $\begin{array}{l}\text { especificas de las } \\
\text { asignaturas }\end{array}$ & $\begin{array}{l}\text { Autoconocimiento y auto- } \\
\text { estima. } \\
\text { Empoderamiento personal. } \\
\text { Reflexión y autoaprendi- } \\
\text { zaje. } \\
\text { Afrontamiento de resulta- } \\
\text { dos. } \\
\text { Confianza. } \\
\text { Motivación para el trabajo. }\end{array}$ & $\begin{array}{l}\text { Implementar proyectos de actuación en di- } \\
\text { versos ámbitos y con diferentes sujetos, im- } \\
\text { pulsando la participación, la formación de } \\
\text { distintos agentes sociales y el seguimiento } \\
\text { reflexivo de las actuaciones para tomar de- } \\
\text { cisiones pertinentes. Comprender los proce- } \\
\text { sos que intervienen en la puesta en práctica } \\
\text { de proyectos. } \\
\text { Identificar los factores y procesos relativos a } \\
\text { la iniciación e implementación de proyectos. }\end{array}$ \\
\hline
\end{tabular}




\begin{tabular}{|c|c|c|}
\hline & Todos Somos Campus & 2. ${ }^{\circ}$ de Educación Social \\
\hline $\begin{array}{l}\text { Competencias } \\
\text { transversales }\end{array}$ & $\begin{array}{l}\text { Actitud cooperativa. Tra- } \\
\text { bajo en equipo. Búsqueda } \\
\text { del bien común. } \\
\text { Respeto por las ideas y } \\
\text { creencias. Reflexión crítica. } \\
\text { Habilidades de comunica- } \\
\text { ción, comunicación inter- } \\
\text { personal, oral y escrita. }\end{array}$ & $\begin{array}{l}\text { Capacidad para trabajar en equipo y para } \\
\text { relacionarse con otras personas del mismo o } \\
\text { distinto ámbito profesional. } \\
\text { Ser capaz de proyectar los conocimientos, } \\
\text { habilidades y destrezas adquiridos para } \\
\text { promover una sociedad basada en los valores } \\
\text { de la libertad, la justicia, la igualdad y el } \\
\text { pluralismo. }\end{array}$ \\
\hline
\end{tabular}

Fuente: Elaboración propia.

\subsubsection{Sesiones de intervención}

Para el desarrollo de la experiencia han sido necesarias 16 sesiones de intervención de una hora aproximada de duración, dichas sesiones fueron desarrolladas durante el segundo cuatrimestre del curso 2018/2019 y se exponen de forma resumida en la Tabla 2, junto con los objetivos a lograr en cada una de ellas:

\section{TABLA 2. Sesiones de intervención desarrolladas}

Sesión 1. Preparación de las actividades.

El objetivo de la sesión ha sido poner en conocimiento de los alumnos de Todos Somos Campus y de segundo de Educación Social el origen de la experiencia y qué pretendemos con esta. Se les explica a ambos grupos (por separado) cómo se van a organizar las siguientes sesiones y se les propone que comiencen con la preparación de una presentación del grupo y la titulación para la siguiente sesión.

Sesión 2. Presentación y formación de grupos.

Con esta sesión se pretende que ambos grupos de alumnos se conozcan. Los alumnos de ambos grupos realizan una presentación con apoyo visual donde se exponen los principales objetivos de sus respectivos programas y que unen esta experiencia: en el caso de los alumnos de Todos Somos Campus formar parte de un programa para el desarrollo de habilidades para el empleo; en el caso de los alumnos de Educación Social, la realización de una práctica en el marco de la asignatura "Procesos y Condiciones para el desarrollo de proyectos", cuyo principal objetivo es promover la planificación y desarrollo de un proyecto de formación para la mejora de la empleabilidad de los educadores sociales. En dicha sesión se exponen también las bases del aprendizaje cooperativo y se forman los grupos de trabajo.

Sesión 3. Habilidades profesionales: dinámica la casa.

El objetivo fundamental de esta sesión es promover el inicio del trabajo en equipo y sentar las bases del desarrollo del proyecto en común; para ello, se pide que cada grupo de trabajo dibuje una casa, y que distribuya la siguiente información:

En la puerta principal: los nombres de los componentes del grupo formado.

En el techo: las expectativas que el grupo tiene puestas en sus respectivos cursos.

En las paredes: las impresiones del grupo sobre su inserción laboral y las capacidades o habilidades profesionales que consideran que pueden demandar las empresas. 


\section{TABLA 2. Sesiones de intervención desarrolladas (cont.)}

Una vez completada la dinámica, se realiza un debate final basado en una "lluvia de ideas" para resaltar las habilidades profesionales más importantes. En el Anexo I podemos encontrar un ejemplo del documento resultante de un grupo de alumnos.

Sesión 4. Realizamos los objetivos de nuestro proyecto de desarrollo de habilidades profesionales para educadores sociales.

Teniendo en cuenta las habilidades fundamentales destacadas en la sesión anterior se comienza la redacción de 5 objetivos específicos del proyecto, por grupo. Tras ello, se realiza la dinámica "bola de nieve" con el fin de llegar a la selección de 4 objetivos para todo el grupo-clase. Sesiones 5 y 6 . Diseño de actividades que nos ayuden a desarrollar dichos objetivos.

En la sesión 5 se realiza en grupos de trabajo una propuesta de ideas para el diseño de actividades para los cinco objetivos. A partir de esta sesión solo continúan los alumnos de Educación Social. Cada objetivo se reparte a uno de los grupos y cada grupo se encarga de concretar las actividades del objetivo que les ha tocado desarrollar.

Sesiones 7, 8, 9, 10, 11,12,13,14. Desarrollo de las actividades.

Los alumnos de Educación Social son los agentes promotores del cambio y receptores al mismo tiempo de este. En estas sesiones son los encargados de desarrollar las actividades diseñadas previamente junto a los estudiantes de TSC. En el Anexo II se muestran las actividades desarrolladas.

Sesiones 15 y 16. Evaluación de la experiencia.

Estas sesiones están destinadas a que tanto alumnos de Todos Somos Campus como alumnos de Educación Social evalúen la experiencia. En siguiente apartado podemos encontrar cómo se realizó y el momento de la evaluación.

Fuente: Elaboración propia.

\subsection{Evaluación de la experiencia}

\subsubsection{Instrumentos para la recogida de información y análisis de datos}

Para la recogida de información se utiliza una metodología mixta apoyada en instrumentos cualitativos (la observación, el grupo de discusión y el análisis de documentos resultantes) y cuantitativos (cuestionario), permitiéndonos obtener información durante todo el proceso al conjugar las posibilidades y ventajas de ambos enfoques (Hernández, Fernández y Baptista, 2007). Dichos instrumentos nos han permitido obtener información procedente de diferentes informantes, permitiéndonos triangular la información, aumentando así su validez.

A continuación, se describe cada uno de estos instrumentos y se explicita cómo ha sido el análisis de la información procedente de estos.

\subsubsection{Cuestionario}

Para la evaluación se ha utilizado un cuestionario estructurado, cuyo objetivo es conocer la perspectiva del alumnado sobre la valoración de la experiencia. Consta de 
diez preguntas cerradas (ítems de puntuación dicotómica: "sí" o "no") y dos preguntas abiertas; la última de ellas dirigida a que los alumnos incorporen las observaciones que estimen oportunas. El cuestionario fue diseñado, por el profesorado participante, en lectura fácil, para mejor comprensión de todo el alumnado participante en la experiencia (ver anexo III) y teniendo en cuenta los requisitos de partida indicados por Ramos, Giménez, Lapaz y Muñoz (2006):

- Sencillez (preguntas breves y sin negaciones o dobles negaciones).

- Brevedad (no demasiados ítems).

- Vocabulario comprensible y adaptado a la edad y características socioculturales de los receptores.

- Motivador y atractivo en el diseño (utilización de letra no demasiado pequeña).

El análisis de datos se llevó a cabo mediante estadísticos descriptivos (frecuencias y porcentajes).

\subsubsection{Registro de observación}

Este instrumento fue utilizado para ayudar a identificar las competencias específicas y transversales desarrolladas por los alumnos. Se trata de un registro de datos formal elaborado a través de observaciones directas no participantes, cumplimentándose por grupo y sesión. Fueron tres los observadores aportando fiabilidad y validez al instrumento pues los resultados eran contrastados entre los distintos observadores (Peña, 2015). El registro de observación aportaba información sobre la organización de los grupos, el clima de trabajo, frecuencia de interacción, comunicación, calidad de las intervenciones, capacidad reflexiva, búsqueda de fuentes de información, motivación hacia el aprendizaje, comportamientos y roles de los miembros, formas de resolución de conflictos, contraste de opiniones, nivel de aceptación de ideas, búsqueda del bien común, registro de incidentes, adecuación de las sesiones, etc. En la tabla siguiente (Tabla 3), se expone dicho registro completo:

\section{TABLA 3. Registro de información en la observación}

\begin{tabular}{|l|l|l|}
\hline Sesión: & Grupo: & Fecha y hora: \\
\hline Dimensión & Aspectos a observar & \multicolumn{1}{|l|}{} \\
\hline Trabajo en equipo & $\begin{array}{l}\text { Organización de los grupos } \\
\text { Rol de los participantes } \\
\text { Liderazgo y colaboración } \\
\text { Clima de trabajo }\end{array}$ \\
& $\begin{array}{l}\text { Frecuencia de interacción } \\
\text { Comportamientos y actitudes }\end{array}$ \\
\hline Comunicación & $\begin{array}{l}\text { Intención comunicativa } \\
\text { Tipo de comunicación (oral o escrita) } \\
\text { Frecuencia y calidad de las intervenciones } \\
\text { Se comparten conocimientos }\end{array}$ \\
\hline
\end{tabular}




\section{TABLA 3. Registro de información en la observación (cont.)}

\begin{tabular}{|l|l|l|}
\hline Sesión: & Grupo: & Fecha y hora: \\
\hline Dimensión & Aspectos a observar \\
\hline $\begin{array}{l}\text { Reflexión crítica y } \\
\text { resolución de conflictos }\end{array}$ & $\begin{array}{l}\text { Contraste de opiniones } \\
\text { Respeto por las ideas de los componentes } \\
\text { Necesidad de contar con las aportaciones de los demás } \\
\text { Incidentes favorables o desfavorables } \\
\text { Resolución de conflictos }\end{array}$ \\
\hline $\begin{array}{l}\text { Motivación hacia el } \\
\text { aprendizaje }\end{array}$ & $\begin{array}{l}\text { Motivación hacia el aprendizaje de los contenidos } \\
\text { Búsqueda y ampliación de información } \\
\text { Actitud activa ante el aprendizaje }\end{array}$ \\
\hline $\begin{array}{l}\text { Organización de las } \\
\text { sesiones }\end{array}$ & $\begin{array}{l}\text { Explicación de las sesiones } \\
\text { Claridad de los objetivos } \\
\text { Adecuación de los espacios } \\
\text { Adecuación del tiempo } \\
\text { Adecuación de la evaluación }\end{array}$ \\
\hline Otras & $\begin{array}{l}\text { Sensaciones percibidas por el docente en relación al trabajo que se } \\
\text { está desarrollando }\end{array}$ \\
\hline
\end{tabular}

Fuente: Elaboración propia.

Para el análisis de la información obtenida a través del registro se realizaban reuniones semanales (desde el inicio de la experiencia hasta el final de esta) donde los observadores intercambian información y llegan a acuerdos sobre el desarrollo de las sesiones y los resultados de aprendizaje de los alumnos. En dichas reuniones se tienen en cuenta, además, los materiales generados por los alumnos a lo largo de todo el proceso educativo.

\subsubsection{Grupo de discusión}

El grupo de discusión fue realizado una vez finalizada la experiencia y sirvió para contrastar la información recogida por los instrumentos anteriores y para recopilar información más detallada respecto a las competencias específicas desarrolladas. En este participaron los alumnos de TSC y de Educación Social, los docentes y la técnica de apoyo. El grupo de discusión se realizó a partir de un guion semiestructurado, elaborado previamente. Las cuestiones formuladas fueron agrupadas en distintos bloques temáticos: valoración de la experiencia, competencias y habilidades desarrolladas y dificultades encontradas. El análisis de datos se realizó a través de la codificación temática aplicada a las transcripciones obtenidas tras la realización del grupo de discusión; estableciendo así un proceso de categorización temática en relación a las respuestas extraídas (Angrosino, 2012; Flick, 2007). 
4. Resultados

\subsection{Resultados del cuestionario: valoración de la experiencia}

Las valoraciones de la experiencia realizadas por los alumnos recogidas a través del cuestionario se muestran en la Tabla 4:

\begin{tabular}{|c|c|c|c|c|}
\hline \multicolumn{5}{|c|}{ TABLA 4. Datos obtenidos del análisis del cuestionario } \\
\hline \multirow{3}{*}{ ÍTEM } & \multicolumn{4}{|c|}{ PORCENTAJE/FRECUENCIAS/MEDIA } \\
\hline & \multicolumn{2}{|c|}{ ESTUDIANTES TSC } & \multicolumn{2}{|c|}{ ESTUDIANTES E. SOCIAL } \\
\hline & Porcentaje & Frecuencia & Porcentaje & Frecuencia \\
\hline ÍTEM 1 & $\begin{array}{l}\text { SÍ } 100 \% \\
\text { NO } \% \%\end{array}$ & $\begin{array}{l}\text { SÍ } 15 \text { alumnos } \\
\text { NO } 0 \text { alumnos }\end{array}$ & $\begin{array}{l}\text { SÍ } 100 \% \\
\text { NO } \% \%\end{array}$ & $\begin{array}{l}\text { SÍ } 30 \text { alumnos } \\
\text { NO } 0 \text { alumnos }\end{array}$ \\
\hline ITEM 2 & $\begin{array}{l}\text { Sí } 100 \% \\
\text { NO } 0 \%\end{array}$ & $\begin{array}{l}\text { SÍ } 15 \text { alumnos } \\
\text { NO } 0 \text { alumnos }\end{array}$ & $\begin{array}{l}\text { Sí } 100 \% \\
\text { NO } \% \%\end{array}$ & $\begin{array}{l}\text { SÍ } 30 \text { alumnos } \\
\text { NO } 0 \text { alumnos }\end{array}$ \\
\hline ITEM 3 & $\begin{array}{l}\text { Sí } 100 \% \\
\text { NO } 0 \%\end{array}$ & $\begin{array}{l}\text { SÍ } 15 \text { alumnos } \\
\text { NO } 0 \text { alumnos }\end{array}$ & $\begin{array}{l}\text { SÍ } 100 \% \\
\text { NO } \% \%\end{array}$ & $\begin{array}{l}\text { SÍ } 30 \text { alumnos } \\
\text { NO } 0 \text { alumnos }\end{array}$ \\
\hline ÍTEM 4 & $\begin{array}{l}\text { SÍ } 100 \% \\
\text { NO } \% \%\end{array}$ & $\begin{array}{l}\text { SÍ } 15 \text { alumnos } \\
\text { NO } 0 \text { alumnos }\end{array}$ & $\begin{array}{l}\text { Sí } 100 \% \\
\text { NO } \% \%\end{array}$ & $\begin{array}{l}\text { SÍ } 30 \text { alumnos } \\
\text { NO } 0 \text { alumnos }\end{array}$ \\
\hline ÍTEM 5 & $\begin{array}{l}\text { SÍ } 100 \% \\
\text { NO } \% \%\end{array}$ & $\begin{array}{l}\text { Sí } 15 \text { alumnos } \\
\text { NO } 0 \text { alumnos }\end{array}$ & $\begin{array}{l}\text { SÍ } 96.67 \% \\
\text { NO } 3.33 \% \\
\end{array}$ & $\begin{array}{l}\text { Sí } 29 \text { alumnos } \\
\text { NO } 1 \text { alumnos }\end{array}$ \\
\hline ITEM 6 & $\begin{array}{l}\text { SÍ } 100 \% \\
\text { NO } \% \%\end{array}$ & $\begin{array}{l}\text { SI } 15 \text { alumnos } \\
\text { NO } 0 \text { alumnos }\end{array}$ & $\begin{array}{l}\text { Sí } 96.67 \% \\
\text { NO } 3.33 \%\end{array}$ & $\begin{array}{l}\text { Sí } 29 \text { alumnos } \\
\text { NO } 1 \text { alumnos }\end{array}$ \\
\hline ITEM 7 & $\begin{array}{l}\text { Sí } 100 \% \\
\text { NO } 0 \%\end{array}$ & $\begin{array}{l}\text { SÍ } 15 \text { alumnos } \\
\text { NO } 0 \text { alumnos }\end{array}$ & $\begin{array}{l}\text { Sí } 100 \% \\
\text { NO } \% \%\end{array}$ & $\begin{array}{l}\text { SÍ } 30 \text { alumnos } \\
\text { NO } 0 \text { alumnos }\end{array}$ \\
\hline ITEM 8 & $\begin{array}{l}\text { SÍ } 100 \% \\
\text { NO } \% \%\end{array}$ & $\begin{array}{l}\text { Sí } 15 \text { alumnos } \\
\text { NO } 0 \text { alumnos }\end{array}$ & $\begin{array}{l}\text { SÍ } 96.67 \% \\
\text { NO } 3.33 \% \\
\end{array}$ & $\begin{array}{l}\text { SÍ } 29 \text { alumnos } \\
\text { NO } 1 \text { alumnos }\end{array}$ \\
\hline ÍTEM 9 & $\begin{array}{l}\text { Sí } 93.33 \% \\
\text { NO } 6.67 \%\end{array}$ & $\begin{array}{l}\text { SÍ } 14 \text { alumnos } \\
\text { NO } 1 \text { alumnos }\end{array}$ & $\begin{array}{l}\text { SÍ } 96.67 \% \\
\text { NO } 3.33 \%\end{array}$ & $\begin{array}{l}\text { SÍ } 29 \text { alumnos } \\
\text { NO } 1 \text { alumnos }\end{array}$ \\
\hline ITEM 10 & $\begin{array}{l}\text { SÍ } 100 \% \\
\text { NO } \% \%\end{array}$ & $\begin{array}{l}\text { Sí } 15 \text { alumnos } \\
\text { NO } 0 \text { alumnos }\end{array}$ & $\begin{array}{l}\text { SÍ } 93.33 \% \\
\text { NO } 6.67 \%\end{array}$ & $\begin{array}{l}\text { Sí } 28 \text { alumnos } \\
\text { NO } 2 \text { alumnos }\end{array}$ \\
\hline ITEM 11 & \multicolumn{2}{|c|}{ MEDIA: 9.7} & \multicolumn{2}{|c|}{ MEDIA: 9.2} \\
\hline
\end{tabular}

Fuente: Elaboración propia.

El cuestionario ha sido cumplimentado por un total de 45 alumnos (15 pertenecientes al programa TSC y 30 estudiantes de Educación Social).

Los resultados obtenidos del análisis de la información aportada por el cuestionario nos indican que la experiencia ha resultado motivante para todos ellos, obteniendo un promedio en su calificación de 9.4 .

Todos los alumnos han destacado que les ha gustado la participación en el aula y la tarea y que la colaboración entre compañeros ha sido buena, el $100 \%$ destaca que los compañeros han escuchado sus opiniones, han resuelto las dudas o le han explicado 
de forma adecuada la actividad. Asimismo, en relación a los compañeros solo un alumno de Educación Social destaca que los compañeros no le han tratado con respeto y que no le han animado a participar.

En cuanto a la labor de los docentes, el $100 \%$ de los alumnos de TSC ha destacado que estos les han animado a participar, solo un alumno de Educación Social destaca que este ítem no se cumple. De la misma forma más del $95 \%$ destaca que los profesores han resuelto las dudas y se han preocupado por su aprendizaje.

En relación a las respuestas de la pregunta abierta del final del cuestionario, los alumnos han destacado que esta experiencia y metodología les ha motivado, ya que han accedido a la información y al aprendizaje de una forma práctica y real, generando de este modo aprendizajes significativos y útiles para su futuro laboral. Asimismo, destacan la importancia de las relaciones establecidas gracias a la experiencia.

\subsection{Resultados de la observación}

Durante el desarrollo de las sesiones no se precisó ninguna incidencia en los equipos de trabajo cooperativo. Todos los participantes de los diferentes grupos aportaron ideas. Asimismo, cuando alguno de los participantes tenía dificultades para entender o desarrollar alguna tarea el resto de miembros del equipo le ayudaban para seguir adelante. Se observa además un aumento de la frecuencia en las interacciones conforme avanzaban las sesiones, así como la mejora de la calidad de las intervenciones y de la motivación hacia el aprendizaje. La resolución de conflictos se desarrolló de forma adecuada, llegando a acuerdos comunes y preocupándose por la búsqueda del bien común. En cuanto a la organización de las sesiones, se observó al principio de estas que no terminaban de quedar claras las explicaciones por lo que se ayudó en las siguientes con apoyos visuales y con la adaptación de materiales haciéndolos accesibles cognitivamente. Asimismo, se encontraron dificultades en los espacios para favorecer el trabajo en equipo y aunque se intentó buscar otros espacios más inclusivos no fue posible acceder a estos.

\subsection{Resultados del grupo de discusión}

En el grupo de discusión los alumnos tanto de Educación Social como del Programa TSC destacan que esta experiencia ha supuesto una oportunidad de interacción entre ambos grupos y les ha permitido aprender unos de otros, tal y como se observa en los siguientes comentarios:

Hemos conocido y hemos podido trabajar con otros estudiantes de la Universidad de Murcia. (A2, TSC)

Hemos aprendido cosas de los compañeros. (A5, TSC)

Compartir opiniones y saber que tenemos un objetivo común, todos hemos aprendido de todos. (A8, ES) 
Asimismo, los alumnos de Educación Social destacan que esta experiencia les ha servido para desmontar prejuicios y cambiar perspectivas y actitudes hacia la discapacidad intelectual:

Me ha encantado la conexión que hemos tenido con los compañeros de Todos Somos Campus, de manera particular he aprendido un montón de cómo son y qué capacidades tienen, ha cambiado mi perspectiva sobre la discapacidad intelectual. (A23, ES)

Todos ellos resaltan que se ha producido un aprendizaje más real, que conecta teoría y práctica, lo que ha provocado un aumento de su motivación hacia el aprendizaje y el desarrollo de competencias específicas y transversales:

Se trata de un aprendizaje real, el hecho de compartir grupos diversos me parece mucho más útil para aprender que cualquier powerpoint o texto. (A30, ES)

Aprendizaje real. (A12, TSC)

La interacción y el compartir los diferentes puntos de vista con objetivo de futuro ha sido una gran motivación. (A10, ES)

Muchos de los participantes destacan que ha mejorado su capacidad de trabajo en equipo, de toma de decisiones y de confianza:

Se han estimulado capacidades como el compañerismo, hemos compartido conocimientos, dudas e ilusiones de algo común como es nuestro futuro laboral. (A22, ES)

Hemos aprendido a trabajar en equipo. (A12, TSC)

Yo me siento más segura a la hora de relacionarme con los demás. (A4, TSC)

Actividades así me ayudan a ser menos tímido y a relacionarme con gente nueva. Habilidades que tengo que tener para trabajar. (A3, TSC)

La comunicación que hemos tenido entre nosotros ha sido muy buena, hemos desarrollado nuestra capacidad de aprendizaje en grupo. (A6, ES)

Además, los docentes destacan mejoras en la responsabilidad y la autoestima de los alumnos:

Están más motivados, observo que tienen y cumplen sus responsabilidades y se sienten más seguros en el contexto universitario. (TE, TSC)

Observo que el hecho de trabajar en común alumnos y alumnas con diferentes capacidades les resulta motivador. Los estudiantes de grado se sienten más responsables en sus propuestas e intervenciones. (D1, TSC, ES)

Asimismo, destacan las habilidades aprendidas respecto de su inserción laboral y el desarrollo de proyectos:

Compartir la actividad con los compañeros, relacionarnos con ellos y las cosas que nos han enseñado sobre inserción laboral. (A7, TSC)

Ya sabemos hacer un proyecto y vamos a hacer un proyecto para irnos de viaje de estudios. (A3, TSC) 
Hemos desarrollado habilidades para poner en práctica en el puesto de trabajo. (A6, ES)

Con metodologías así aprendemos realmente a implementar proyectos. (A22, ES)

En relación a las dificultades encontradas todos los participantes destacan la falta de tiempo en la realización de las actividades, sobre todo para la participación de los alumnos de TSC en el desarrollo de las actividades.

Ha faltado tiempo para la realización de las actividades. (A1, TSC)

Es cierto que la experiencia habría sido aún más enriquecedora si hubiésemos dispuesto de más tiempo con los alumnos de Todos Somos Campus en el desarrollo del proyecto. (D2, ES)

\section{Discusión y conclusiones}

Como conclusión podemos establecer que los alumnos participantes en la experiencia valoran muy positivamente esta, tanto para alcanzar sus aprendizajes como para desarrollar competencias específicas y transversales.

Al ser protagonistas de su propio aprendizaje se realizan aprendizajes más significativos, son los propios alumnos quienes investigan, van a las fuentes de información y se preocupan por conocer la realidad. La responsabilidad de las actividades deja de estar centrada únicamente en el docente y pasa a ser compartida por todos los participantes. Esta situación favorece el control autónomo de los aprendizajes, la mejora de la capacidad resolutiva y un aumento de la responsabilidad ante la toma de decisiones.

La mayor dificultad radica en que los alumnos no suelen trabajar con este tipo de enfoques y al tratarse de estrategias desconocidas requieren de más tiempo.

Nuestros resultados confirman los resultados obtenidos por autores como Oberto (2014) y Cerrillo, Izuzquiza y Egido (2013), ya que podemos concluir que el aprendizaje cooperativo entre alumnos con discapacidad intelectual y otros miembros de la comunidad universitaria es importante porque promueve mejoras tanto en las capacidades académicas como en las habilidades relacionadas con el desarrollo personal y social:

a) Permite al alumnado desarrollar habilidades emocionales y sociales, sobre todo se produce una mejora de la motivación y la autoestima. En el caso de los alumnos participantes de Todos Somos Campus, el mero hecho de formar parte de las actividades habituales para personas de su edad y participar en el entorno universitario aumenta su confianza.

b) Aumenta el rendimiento estudiantil y asegura el logro de los objetivos de la instrucción. Además, se provoca una mejora en habilidades académicas como la capacidad comunicativa.

c) Los beneficios no se limitan a los alumnos receptores, se constatan efectos positivos para todos los colectivos implicados y beneficia a la sociedad en general ya que crea ciudadanos dispuestos a trabajar en equipo para crear una universidad y sociedad mejor. 
Por tanto, generar espacios de aprendizaje entre personas con y sin discapacidad aporta beneficios a todos los implicados y genera la posibilidad de crear aulas heterogéneas que busquen una verdadera inclusión educativa y que huyan de la segregación; pues esta última tiene lugar cuando la educación de alumnos con discapacidad se imparte en entornos separados, diseñados exclusivamente para atender unas necesidades específicas y apartándolos del resto de alumnos sin discapacidad (Comité sobre los Derechos de las Personas con Discapacidad, 2014). Por ello, experiencias como la descrita son el camino hacia una verdadera inclusión educativa.

No obstante, y a pesar de los buenos resultados obtenidos, encontramos una serie de limitaciones, las cuales deben ser consideradas para posteriores trabajos:

- La situación de partida de los alumnos solo es evaluada a través de la observación, por lo que sería adecuado utilizar otro instrumento de evaluación que fuese cumplimentado al inicio y al final de la experiencia para aportar mayor veracidad a la investigación, ya que facilitaría la triangulación de la información y el establecimiento de conclusiones. Por tanto, sería necesario aplicarlo antes de cualquier tipo de intervención, para identificar el nivel de partida real de los alumnos y al final de esta para identificar claramente los logros conseguidos.

- Los instrumentos, elaborados ad hoc, no cuentan con una validación y fiabilidad revisada, por tanto, y para aportar mayor consistencia metodológica a la evaluación este es un paso a realizar.

- El tiempo en el que se desarrolló la experiencia es limitado. Ampliar su desarrollo es fundamental para la obtención de mejores resultados, si cabe, y para favorecer el desarrollo de la cooperación e inclusión del alumnado.

\section{Referencias bibliográficas}

Angrosino, M. (2012). Etnografía y observación participante en investigación cualitativa. Madrid: Morata.

Azorín, C. (2018). El método de aprendizaje cooperativo y su aplicación en las aulas. Perfiles Educativos, 40(161), 181-194.

CDPD - Comité de Derechos de las Personas con Discapacidad (2016). Observación General núm. 4 (2016) sobre el derecho a la educación inclusiva. (CRPD/C/GC/4). Ginebra: Naciones Unidas.

Cerrillo, R., Izuzquiza, D. y Egido, I. (2013). Inclusión de jóvenes con discapacidad intelectual en la Universidad. Revista de Investigación en Educación, 11(1), 41-57.

Domingo, J. (2010). El aprendizaje cooperativo y las competencias. Revista d'Innovació Docent Universitària, 2, 1-9.

Estrada, M., Monferrer, D. y Moliner, M. (2016). El aprendizaje cooperativo y las habilidades socioemocionales: una experiencia docente en la asignatura Técnicas de Ventas. Formación Universitaria, 9(6), 43-62.

Flick, U. (2007). Introducción a la investigación cualitativa. Madrid: Morata.

Florido, C., Jiménez, J. L., y SAntana, I. (2011). Obstáculos en el camino hacia Bolonia: efectos de la implantación del Espacio Europeo de la Educación Superior (EEES) sobre los resultados académicos. Revista de Educación, 354, 629-656. 
García-Ruiz, R., Guerra, S., González, N. y Álvarez, E. (2010). Estudio exploratorio de las percepciones del profesorado universitario respecto a la gestión de la docencia. Educación XX1, 13(2), 163-184.

GutiérRez-Fresneda, R. (2017). La opinión de los/as estudiantes sobre el aprendizaje cooperativo para la mejora de la práctica docente. En Investigación en docencia universitaria: diseñando el futuro a partir de la innovación educativa (pp. 548-553). Barcelona: Octaedro.

Hart, D., Grigal, M., Sax, C., Martínez, D. y Will, M. (2006). Postsecondary education options for students with intellectual disabilities. Research to Practice, 45.

Hernández, R., Fernández, C. y Baptista, P. (2007). Metodología de la investigación. México: McGraw-Hill.

IzuzQuiza, D. y Rodríguez, P. (2016). Evaluación de la metodología empleo con apoyo (ECA), en el Programa Promentor (UAM-PRODIS). El ajuste competencial. Siglo Cero, 47(1), 37-54. http://dx.doi.org/10.14201/scero201613754.

Johnson, D. y Johnson, R. (2014). La evaluación en el aprendizaje cooperativo. Cómo mejorar la evaluación individual a través del grupo. Madrid: Ediciones SM.

Johnson, R. y Johnson, D. (2009). Joining together: group theory and group skills. Boston: Allyn and Bacon.

Lata, S. y CAstro, M. (2016). El aprendizaje cooperativo, un camino hacia la inclusión educativa. Revista Complutense de Educación, 27(3), 1085-1101.

León del Barco, B., Mendo, S., Felipe, E., Polo, M. I. y Fajardo, F. (2017). Potencia de equipo y aprendizaje cooperativo en el ámbito universitario. Revista de Psicodidáctica, 22(1), 9-15.

OberTo, T. (2014). El aprendizaje cooperativo como herramienta para la educación universitaria. Revista Educación en Valores, 1(21), 58-69.

Ochoa, A., Pérez, L. M. y Salinas, J. (2018). El aprendizaje-servicio (APS) como práctica expansiva y transformadora. Revista Iberoamericana de Educación, 76, 15-34.

Palomares, A. (2009). El nuevo modelo docente en el paradigma formativo centrado en el alumno. Enseñanza and Teaching, 27(2), 45-75.

Peña, A. B. (2015). La observación como herramienta científica. Madrid: ACCI (Asociación Cultural y Científica Iberoamericana).

Pujolás, P. (2012). Aulas inclusivas y aprendizaje cooperativo. Educatio Siglo XXI, 30(1), 89-112.

Ramos, R., Giménez, A. I., Lapaz, E. y Muñoz, M. A. (2006). Cuestionario de evaluación de la autoestima para educación primaria. Madrid: TEA Ediciones.

RierA, G. (2011). El aprendizaje cooperativo como metodología clave para dar respuesta a la diversidad del alumnado desde un enfoque inclusivo. Revista Latinoamericana de Educación Inclusiva, 5(2), 133-149.

Salmerón, C. (2010). Desarrollo de la competencia social y ciudadana a través del aprendizaje cooperativo. Tesis doctoral. Universidad de Granada.

Slavin, R. (2014). Cooperative Learning and Academic Achievement: Why does groupwork work? Anales de Psicología, 30(3), 785-791.

Torrego, J. C. y Negro, A. (2012). El aprendizaje cooperativo en las aulas. Madrid: Alianza Editorial.

Trujillo, F. y Ariza, M. Á. (2006). Experiencias educativas en aprendizaje cooperativo. Granada: Grupo Editorial Universitario. 


\section{ANEXO I}

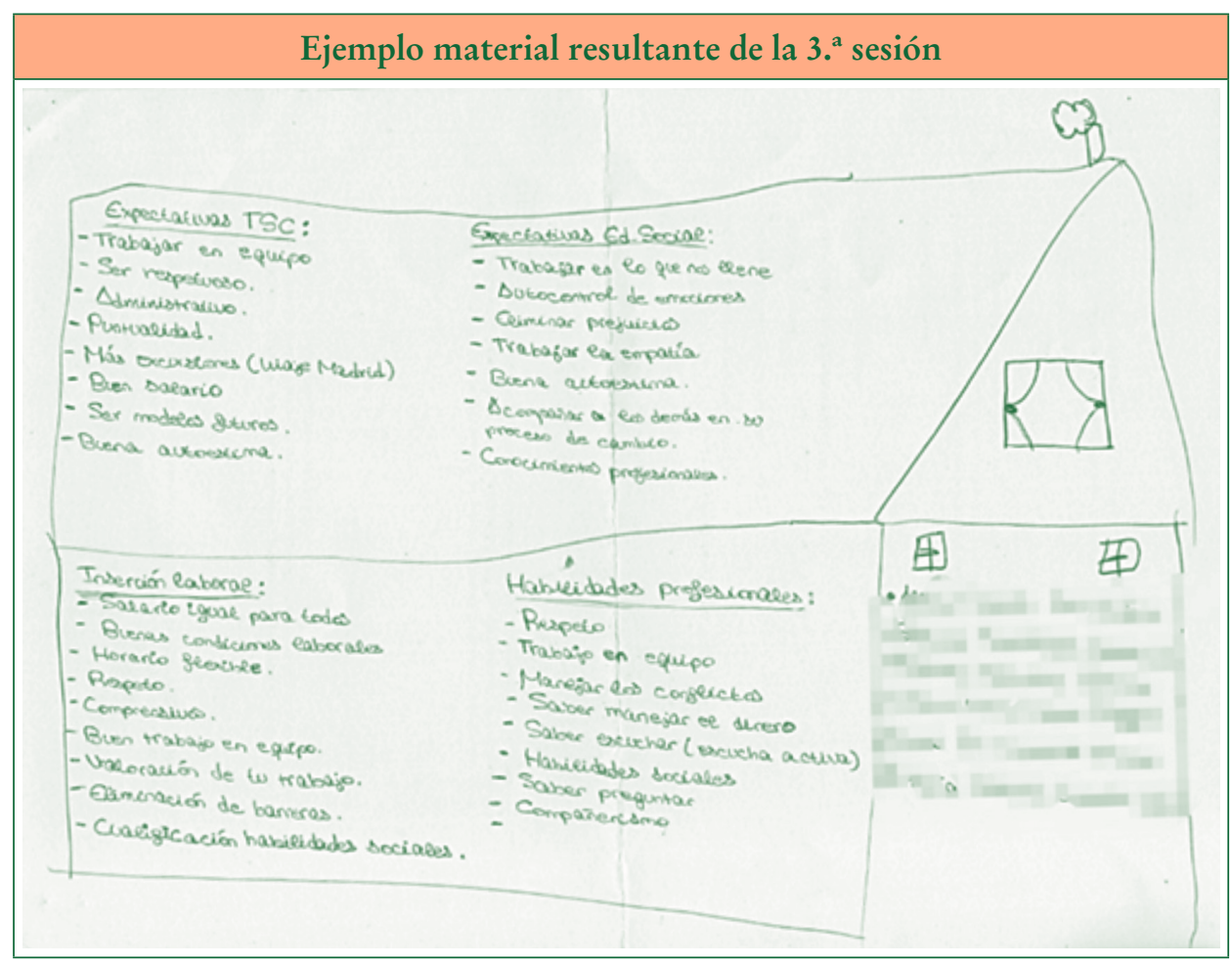

Ediciones Universidad de Salamanca / CC BY-NC-ND

Siglo Cero, vol. 51 (2), 2020, abril-junio, pp. 55-72 


\section{Objetivos y actividades desarrolladas durante las sesiones 7-14}

Objetivo general. Orientar al alumno para que consiga de forma activa su inserción laboral: formando, capacitando y motivando a los alumnos a desarrollar actitudes dinámicas en el proceso de búsqueda de empleo.

Objetivo específico 1. Identificar las diferentes salidas respecto al mercado laboral y ámbitos de actuación del educador social.

Actividades.

- Realización de actividades por áreas en las que puede trabajar un educador social (bullying, animación sociocultural, atención a la diversidad, violencia de género...). Se trata de una serie de actividades que ayudan a conocer en mayor profundidad al colectivo y empatizar con este.

Objetivo específico 2. Desarrollar habilidades sociales y emocionales para una adecuada incorporación al mercado laboral (promover habilidades para evitar que el trabajo afecte en la vida personal).

Actividades.

- Dinámica "miedos profesionales”. Esta actividad consiste en analizar los miedos que los participantes sienten a la hora de afrontar un futuro en el mercado laboral, trabajando con el colectivo que desean.

- Cubo de las emociones. Esta dinámica consiste en relatar situaciones de la vida personal de cada educador/a social. Cada cara del cubo lleva inscrita una emoción: impotencia, inseguridad, satisfacción, miedo, soledad y agradecimiento.

Objetivo específico 3. Adquirir conocimientos respecto a técnicas e instrumentos de búsqueda de empleo para el desenvolvimiento en el mercado de trabajo.

Actividades.

- Dinámica "círculo contrario". Dinámica cuyo objetivo es demostrar la importancia del cuerpo en los actos comunicativos.

- Juego de roles relacionado con la buena comunicación y técnicas de persuasión.

- Dinámica con post it para la selección de las competencias más importantes para incluir en un currículum.

- Elaboración de un currículum alternativo.

- Presentación de diferentes recursos para la búsqueda de empleo (aplicaciones, INEM, SEF CARM).

- Simulación de entrevista de trabajo (roleplaying).

Objetivo específico 4. Fomentar el trabajo en equipo y desarrollar estrategias para la resolución de conflictos.

Actividades.

- Dinámica "el río", "el corro musical" y "la isla desierta”. Dinámicas para el fomento del trabajo en equipo que muestran que el trabajo en grupo obtiene mejores resultados que el individual.

- Dinámica "verdades y mentiras". Dinámica que ayuda a impedir la formación de prejuicios.

- Dinámica "ganador/perdedor". Dinámica que ayuda a identificar el lado positivo de las experiencias negativas.

- Dinámica "telaraña adaptada” y "la bandera”. Dinámicas que pretenden fomentar las habilidades de resolución de conflictos y la búsqueda de soluciones comunes de un equipo ante una situación problemática.

- Dinámica "el sí y el no". Dinámica que se lleva a cabo con la pretensión de desarrollar la flexibilidad de opiniones, promover el acercamiento de distintas posiciones sobre el trabajo en equipo y la resolución de conflictos y conocer y reflexionar sobre cómo cada uno de los participantes se muestra al resolver un conflicto y al trabajar en equipo. 


\begin{tabular}{|c|c|c|}
\hline \multicolumn{3}{|c|}{ Cuestionario elaborado para evaluar la experiencia desarrollada } \\
\hline \multicolumn{3}{|l|}{ CUESTIONARIO DE EVALUACIÓN ACTIVIDAD } \\
\hline \multicolumn{3}{|l|}{$\begin{array}{l}\text { NOMBRE Y APELLIDOS DEL ALUMNO/A } \\
\text { FECHA: }\end{array}$} \\
\hline \multicolumn{3}{|c|}{ Responde a las siguientes preguntas marcando con una $\mathrm{X}$ lo que se indica } \\
\hline 1. La participación en el aula me ha gustado & $\begin{array}{c}\text { Sí } \\
1 \\
\end{array}$ & $\begin{array}{l}\mathrm{NO} \\
0\end{array}$ \\
\hline 2. Mis compañeros y compañeras me han explicado bien la actividad & $\begin{array}{c}\text { Sí } \\
1 \\
\end{array}$ & $\begin{array}{l}\mathrm{NO} \\
0\end{array}$ \\
\hline 3. Mis compañeros y compañeras han escuchado mis opiniones & \begin{tabular}{|c|} 
Sí \\
$L$ \\
\end{tabular} & $\begin{array}{l}\mathrm{NO} \\
0\end{array}$ \\
\hline 4. Mis compañeros y compañeras han respondido siempre a mis dudas & $\begin{array}{c}\text { Sí } \\
L \\
\end{array}$ & $\begin{array}{l}\mathrm{NO} \\
0\end{array}$ \\
\hline 5. Mis compañeros y compañeras me han animado a participar & $\begin{array}{c}\text { Sí } \\
L \\
\end{array}$ & $\begin{array}{l}\mathrm{NO} \\
0\end{array}$ \\
\hline 6. Mis compañeros y compañeras me han tratado con respeto & $\begin{array}{c}\text { Sí } \\
\text { L } \\
\end{array}$ & $\begin{array}{l}\mathrm{NO} \\
9\end{array}$ \\
\hline 7. Las tareas me han gustado & $\begin{array}{c}\text { Sí } \\
1 \\
\end{array}$ & $\begin{array}{l}\mathrm{NO} \\
0\end{array}$ \\
\hline 8. Los profesores me han animado a participar & $\begin{array}{c}\text { Sí } \\
1 \\
\end{array}$ & $\begin{array}{l}\text { NO } \\
0\end{array}$ \\
\hline 9. Los profesores han resuelto mis dudas & $\begin{array}{c}\text { Sí } \\
1 \\
\end{array}$ & $\begin{array}{l}\mathrm{NO} \\
9\end{array}$ \\
\hline 10. Los profesores se han preocupado de que aprenda & $\begin{array}{c}\text { SÍ } \\
1 \\
\end{array}$ & $\begin{array}{l}\mathrm{NO} \\
0\end{array}$ \\
\hline 11. ¿Qué nota le pondrías a las actividades realizadas? Del 0 al 10 & & \\
\hline Di qué cosas te han gustado más y cuáles te han gustado menos & & \\
\hline
\end{tabular}

